\title{
A simple model of economic geography à la Helpman-Tabushi
}

Y. Murata and J. F. Thisse

Discussion Paper 2005-17

\section{Département des Sciences Économiques de l'Université catholique de Louvain}




\title{
A simple model of economic geography à la Helpman-Tabuchi*
}

\author{
Yasusada Murata ${ }^{\dagger}$ and Jacques-François Thisse ${ }^{\ddagger}$ \\ First Version: September 14, 2004 \\ Revised Version: February 15, 2005
}

\begin{abstract}
This paper explores the interplay between commodities' transportation costs and workers' commuting costs within a general equilibrium framework à la Dixit-Stiglitz. Workers are mobile and choose a region where to work as well as an intraurban location where to live. We show that a more integrated economy need not be more agglomerated. Instead, low transportation costs lead to the dispersion of economic activities. This is because workers are able to alleviate the burden of urban costs by being dispersed, while retaining a good access to all varieties. By contrast, low commuting costs foster the agglomeration of economic activities.
\end{abstract}

Keywords: commuting costs, urban costs, transportation costs, economic geography, agglomeration

JEL classification: F12, R12

\footnotetext{
*We would like to thank Jan Brueckner, two referees, Kristian Behrens, Carl Gaigné and Frédéric Robert-Nicoud for valuable comments and suggestions. Part of this paper was written while the first author was visiting CORE. The usual disclaimer applies.

${ }^{\dagger}$ Faculty of Economics, Tokyo Metropolitan University, 1-1 Minami-Ohsawa, Hachioji, Tokyo 192-0397 (Japan)

${ }^{\ddagger}$ CORE, Université catholique de Louvain, Voie du Roman Pays 34, 1348 Louvain-la-Neuve (Belgium), PSE (France) and CEPR.
} 


\section{Introduction}

Ever since the pioneering work of Krugman [9], the core-periphery model has been criticized because it does not fit well contemporary space-economies. First, nowadays, the main dispersion force seems to lie in the existence of urban costs, defined as the sum of housing and commuting costs, borne by workers living in large agglomerations, and not in the agricultural sector whose share in employment and expenditure has sharply decreased in most industrialized countries. Second, Krugman's model fails to recognize that economic agglomerations typically generate higher costs to be paid by residents when the population size rises. Yet, such costs are unavoidable once agglomeration takes the form of a city.

In this perspective, two papers are worth mentioning. Firstly, Tabuchi [15] may be viewed as an attempt to unify urban economics à la Alonso [1] and new economic geography. Indeed, his model allows for the interplay between commuting costs and transportation costs in a spatial economy. Unfortunately, however, his analysis has a significant shortcoming: analytical results are available only for the two extreme cases of zero and infinite transportation costs. Independently, Helpman [7] has introduced a housing market into an economic geography model in which all workers are mobile. However, cities have no spatial extension in his setting because Helpman abstracts from commuting costs. In addition, his treatment of the model is purely numerical.

The aim of this paper is to propose a simple model of economic geography integrating both transportation and commuting costs when labor is homogeneous and mobile. As usual, the agglomeration force finds its origin in the need to reduce transportation costs of manufactured goods, but the main dispersion forces now stem from land consumption and the resulting need for workers to commute. While retaining the general equilibrium framework of monopolistic competition à la Dixit-Stiglitz [4], we introduce iceberg commuting costs, as in Krugman and Livas Elizondo [10] or Duranton and Puga [5], together with the standard iceberg transportation costs of economic geography. ${ }^{1}$ The use of the same modeling strategy for both types of spatial costs presents several advantages.

In this respect, the following results stand out. First, unlike Krugman [9], agglomeration is a stable equilibrium when transportation costs are large but dispersion prevails when they are low. Second, agglomeration is always a stable equilibrium once commuting costs are sufficiently low. Third, whereas there always exists a sustain point (i.e. a level of transportation costs above which agglomeration is a stable equilibrium), a break point (i.e. a level of transportation costs above which dispersion is unstable) may not exist. Fourth, we work with a Dixit-Stiglitz-iceberg model that is "almost" analytically solvable by means of paper and pencil. In particular, we are able to determine the exact analytical expressions for both the break and sustain points, thus making their comparison easier.

\footnotetext{
${ }^{1}$ Note that Tabuchi and Thisse [16] develop a similar approach using a completely different modeling strategy: the profit-maximizing price varies with the mass of competing firms, but there is no income effect in consumer demand (see Ottaviano et al. [12]).
} 
Interestingly, we may go one step further by determining the break and sustain points in terms of commuting costs, which are the counterpart of the standard break and sustain points. However, a complete characterization of the set of equilibria is out of reach. Last, we uncover a new effect: because workers save time on commuting when there is dispersion, more labor is available for production, thus implying a larger total mass of varieties at the symmetric equilibrium than when agglomeration prevails. This new dispersion force captures (although indirectly) the well-known idea that the formation of large agglomerations takes resources away from private consumption because of the construction of major urban infrastructures.

Our results thus suggest that a more integrated economy need not be more agglomerated. Quite the opposite: we show that low transportation costs lead to the dispersion of economic activities because this allows workers to alleviate the burden of urban costs. In accord with what economic historians have observed (see, e.g. Bairoch [3]; Hohenberg and Lees [8]), the agglomeration is more likely to arise when commuting costs within cities get smaller and smaller. In this respect, it is worth noting that if the process of globalization affects the shipping costs of commodities, it has no direct impact on workers' commuting costs. Globalization need not, therefore, lead to a more polarized economic space. Instead, it might well favor a more dispersed space-economy.

The model is introduced in Section 2. The properties of the spatial equilibrium are derived in Section 3, whereas Section 4 concludes.

\section{The model}

\subsection{The spatial economy}

Consider an economy involving two regions (labelled $r=1,2$ ), one industrial sector producing a differentiated product by using labor as its sole input, and two goods (the differentiated product and land). The economy is endowed with a unit mass of identical and mobile workers, as well as with a large amount of land in each region. Each worker owns one unit of labor. Let $\lambda$ denote the fraction of workers residing in region 1 so that the mass of workers in regions 1 and 2 is respectively given by $L_{1}=\lambda$ and $L_{2}=1-\lambda_{.}{ }^{2}$

The welfare of a worker depends on her consumption of the two goods. The first good is supplied as a continuum of varieties of a horizontally differentiated good, produced under monopolistic competition and increasing returns. Any variety of this good can be shipped from one region to the other according to an iceberg transportation technology: $T>1$ units of the variety must be sent from the origin for one unit to arrive at destination; $T$ thus accounts for all the impediments to trade.

\footnotetext{
${ }^{2}$ It would be easy to expand our setting by allowing either a fraction of the labor force to be immobile or the workers to be heterogeneous in their perception of urban features, for each region to retain a minimum positive population size.
} 
The second good is land and is perfectly immobile. Each region is formed by a city spread along a one-dimensional space $X$. The amount of land available at each location $x \in X$ is equal to one. All firms located in region $r$ are set up at the Central Business District (in short CBD) situated at the origin $x=0$ of $X$.

Each worker consumes one unit of land, supplies one unit of labor, and commutes to the CBD. Hence, in equilibrium, workers are equally distributed around the CBD of region $r$ whose urban landscape is therefore given by $\left[-L_{r} / 2, L_{r} / 2\right]$. We assume that commuting costs have the nature of an iceberg, thus implying that the effective labor supply by a worker living at a distance $|x|$ from the CBD is given by

$$
s(x)=1-2 \theta|x| \quad x \in\left[-L_{r} / 2, L_{r} / 2\right]
$$

where $\theta>0$ captures the efficiency loss due to commuting. For $s(x)$ to be positive regardless of the spatial distribution of workers, we assume $\theta<1$ throughout the paper. As a result, the total effective labor supply in region $r$ is given by

$$
S_{r}=\int_{-L_{r} / 2}^{L_{r} / 2} s(x) d x=L_{r}\left(1-\theta L_{r} / 2\right) .
$$

We normalize the land rent at both city edges at zero. Then, if $w_{r}$ stands for the wage rate paid to the workers by the firms at the CBD of region $r$, the wage net of commuting costs earned by a worker residing at either edge is such that:

$$
s\left(-L_{r} / 2\right) w_{r}=s\left(L_{r} / 2\right) w_{r}=\left(1-\theta L_{r}\right) w_{r} .
$$

Because workers are identical, the wage net of both commuting costs and land rent must be equal across all locations. Thus, it must be that $s(x) w_{r}-R_{r}(x)=s\left(-L_{r} / 2\right) w_{r}=s\left(L_{r} / 2\right) w_{r}$ where $R_{r}(x)$ is the land rent prevailing in region $r$ at a distance $|x|<L_{r} / 2$ from its CBD. Then, for a given distribution of workers across regions, the equilibrium land rent in region $r$ is given by

$$
R_{r}^{*}(x)=\theta\left(L_{r}-2|x|\right) w_{r} .
$$

The aggregate land rent in region $r$ is then equal to

$$
A L R_{r}=\int_{-L_{r} / 2}^{L_{r} / 2} R_{r}^{*}(x) d x=\theta L_{r}^{2} w_{r} / 2 .
$$

It remains to describe how this aggregate land rent is distributed. We consider each region as an independent jurisdiction that owns the land of its region only. This is a reasonable assumption as long as there exists no "global government". As a result, each worker living in region $r$ owns an equal share of land in her region of residence. Accordingly, in addition to her wage, each worker receives an income $A L R_{r} / L_{r}=\theta L_{r} w_{r} / 2$ from her land ownership. ${ }^{3}$

\footnotetext{
${ }^{3}$ Tabuchi [15] assumes absentee landlords, whereas there is global land ownership in Helpman [7].
} 


\subsection{Consumption}

Regarding the consumption of the differentiated good, each worker in region $r$ maximizes a CES-utility function given by

$$
U_{r}=\left[\int_{i \in \mathcal{I}_{r}} c_{r r}(i)^{\frac{\sigma-1}{\sigma}} d i+\int_{i \in \mathcal{I}_{s}} c_{r s}(i)^{\frac{\sigma-1}{\sigma}} d i\right]^{\frac{\sigma}{\sigma-1}}
$$

subject to the budget constraint

$$
\begin{aligned}
\int_{i \in \mathcal{I}_{r}} p_{r}(i) c_{r r}(i) d i+\int_{i \in \mathcal{I}_{s}} p_{s}(i) T c_{r s}(i) d i & =\left(1-\theta L_{r}\right) w_{r}+\theta L_{r} w_{r} / 2 \\
& =\left(1-\theta L_{r} / 2\right) w_{r}
\end{aligned}
$$

where $\mathcal{I}_{r}$ is the set of varieties produced in region $r$. Then, her consumption for each variety is

$$
\begin{aligned}
& c_{r r}(i)=p_{r}(i)^{-\sigma} P_{r}^{\sigma-1}\left(1-\theta L_{r} / 2\right) w_{r} \\
& c_{r s}(i)=p_{s}(i)^{-\sigma} T^{-\sigma} P_{r}^{\sigma-1}\left(1-\theta L_{r} / 2\right) w_{r}
\end{aligned}
$$

where the price index in region $r$ is given by

$$
P_{r}=\left[\int_{i \in \mathcal{I}_{r}} p_{r}(i)^{1-\sigma} d i+\int_{i \in \mathcal{I}_{s}} p_{s}(i)^{1-\sigma} T^{1-\sigma} d i\right]^{\frac{1}{1-\sigma}} .
$$

It is then readily verified that her indirect utility is as follows:

$$
V_{r}=\frac{\left(1-\theta L_{r} / 2\right) w_{r}}{P_{r}}
$$

Workers are attracted by the region that yields the higher utility level so that their mobility may be described by the following adjustment process:

$$
\dot{L}_{r}=\left(V_{r}-\bar{V}\right) L_{r}
$$

where $\bar{V} \equiv V_{1} L_{1}+V_{2} L_{2}$. Clearly, migrations stop once $L_{r}=0$ in one region.

\subsection{Production}

The labor input requirement for producing $y_{r}$ units of variety $i$ is given by

$$
l_{r}(i)=F+v y_{r}(i)
$$

where $F$ and $v$ stand for the fixed and marginal labor inputs, respectively. Given the above consumer demand, each firm maximizes its profit

$$
\pi_{r}(i)=p_{r}(i) y_{r}(i)-w_{r}\left[F+v y_{r}(i)\right]
$$


where $y_{r}(i)$ is given by

$$
y_{r}(i)=c_{r r}(i) L_{r}+T c_{s r}(i) L_{s}
$$

It is well known that firm $i$ 's profit-maximizing price has the following form:

$$
p_{r}^{*}(i)=\frac{\sigma v}{\sigma-1} w_{r} .
$$

Recalling that $v$ stands for the number of labor units needed to produce one unit of the differentiated product, without loss of generality we may then choose the unit of this good for $v$ to satisfy the condition $\sigma v /(\sigma-1)=1$, so that $p_{r}^{*}(i)=w_{r}$.

Finally, the zero-profit condition yields $y_{r}^{*}(i)=\sigma F$. In what follows, we choose the unit of labor such that $\sigma F=1$ so that the profit-maximizing output of a firm becomes $y_{r}^{*}(i)=1$. It then follows from our two normalization rules that

$$
l_{r}^{*}(i)=\frac{1}{\sigma}+v=\frac{1}{\sigma}+\frac{\sigma-1}{\sigma}=1 .
$$

\section{The spatial equilibrium}

\subsection{Preliminary results}

Let $N_{r}$ be the mass of firms located in region $r$. The labor market equilibrium condition in region $r$ is given by

$$
\int_{0}^{N_{r}} l_{r}^{*}(i) d i=S_{r}
$$

so that $N_{r}=S_{r}$. Then, we have the following relationship between the total mass of varieties and the spatial distribution of workers.

Proposition 1. The more symmetric the spatial distribution of workers, the larger the total mass of varieties in the economy.

Proof. Differentiating the total mass of varieties with respect to $\lambda$, we have

$$
\begin{gathered}
\frac{\partial\left(N_{1}+N_{2}\right)}{\partial \lambda}=\frac{\partial\left(S_{1}+S_{2}\right)}{\partial \lambda}=\theta(1-2 \lambda) \\
\frac{\partial^{2}\left(N_{1}+N_{2}\right)}{\partial \lambda^{2}}=\frac{\partial^{2}\left(S_{1}+S_{2}\right)}{\partial \lambda^{2}}=-2 \theta<0 .
\end{gathered}
$$

Thus, the total mass of varieties is maximized at $\lambda=1 / 2$ and declines as $\lambda$ increases or decreases from $\lambda=1 / 2$. 
Intuitively, when the economy is dispersed, commuting costs are lower, thus implying that more labor is available for the industrial sector. The fact that the total mass of varieties varies with the spatial distribution of workers makes our model more general than the existing ones in which the total number of varieties is constant regardless of the spatial distribution of firms. ${ }^{4}$ More precisely, Proposition 1 shows that agglomeration generates two types of costs for the workers: higher urban costs as well as a narrower range of varieties. It is worth stressing that this result is obtained in the absence of any technological externality.

The market clearing conditions for the differentiated product are as follows:

$$
\begin{aligned}
& c_{11}(i) L_{1}+T c_{21}(i) L_{2}=1 \\
& T c_{12}(i) L_{1}+c_{22}(i) L_{2}=1 .
\end{aligned}
$$

These equations yield the two wage equations:

$$
\begin{aligned}
& w_{1}^{\sigma}=P_{1}^{\sigma-1} S_{1} w_{1}+T^{1-\sigma} P_{2}^{\sigma-1} S_{2} w_{2} \\
& w_{2}^{\sigma}=T^{1-\sigma} P_{1}^{\sigma-1} S_{1} w_{1}+P_{2}^{\sigma-1} S_{2} w_{2}
\end{aligned}
$$

where the price indices can be rewritten as

$$
\begin{aligned}
& P_{1}=\left[S_{1} w_{1}^{1-\sigma}+S_{2}\left(w_{2} T\right)^{1-\sigma}\right]^{\frac{1}{1-\sigma}} \\
& P_{2}=\left[S_{1}\left(w_{1} T\right)^{1-\sigma}+S_{2} w_{2}^{1-\sigma}\right]^{\frac{1}{1-\sigma}} .
\end{aligned}
$$

As in Murata [11], for any given $\lambda$, hence $S_{1}$ and $S_{2}$, the above four equations can be shown to have a unique solution for $\left\{P_{1}, P_{2}, w_{1}, w_{2}\right\}$.

\subsection{The interplay between transportation and commuting costs}

In this model, urban costs act as a dispersion force through workers' income, whereas transportation costs generate an agglomeration force through the price index. The argument involves three steps.

First, let $\omega \equiv w_{1} / w_{2}$ and $\varepsilon \equiv S_{1} /\left(S_{1}+S_{2}\right)$. For any given value of $\varepsilon, \omega$ is implicitly determined from either of the two wage equations by means of the following expression:

$$
\varepsilon=\frac{1}{1+\frac{\omega^{1-2 \sigma}-\omega^{1-\sigma} T^{1-\sigma}}{1-\omega^{-\sigma} T^{1-\sigma}}} .
$$

Murata [11] shows that $\varepsilon$ is strictly increasing in $\omega$ over the interval for which $\varepsilon \in(0,1)$, so that there exists an inverse function $\omega(\varepsilon)$, which is also strictly increasing.

\footnotetext{
${ }^{4}$ In a special, but analytically solvable, version of the economic geography model with vertical linkages, Ottaviano and Robert-Nicoud [13] show that the total mass of varieties is larger at the core-periphery equilibrium than at the symmetric equilibrium.
} 
Second, we determine the relative utility across regions:

$$
\begin{aligned}
\frac{V_{2}[\lambda, \varepsilon(\lambda), \omega(\varepsilon(\lambda))]}{V_{1}[\lambda, \varepsilon(\lambda), \omega(\varepsilon(\lambda))]} & =\frac{1-\theta(1-\lambda) / 2}{1-\theta \lambda / 2} \frac{w_{2} / P_{2}}{w_{1} / P_{1}} \\
& =\underbrace{\frac{1-\theta(1-\lambda) / 2}{1-\theta \lambda / 2}}_{\mathcal{U}(\lambda)}[\underbrace{\left[\frac{\varepsilon(\lambda)+(1-\varepsilon(\lambda)) T^{1-\sigma} \omega(\varepsilon(\lambda))^{\sigma-1}}{\varepsilon(\lambda) \omega(\varepsilon(\lambda))^{1-\sigma} T^{1-\sigma}+(1-\varepsilon(\lambda))}\right]^{\frac{1}{1-\sigma}}}_{\mathcal{T}(\lambda)}
\end{aligned}
$$

where

$$
\varepsilon(\lambda)=\frac{S_{1}(\lambda)}{S_{1}(\lambda)+S_{2}(\lambda)}=\frac{\lambda(2-\theta \lambda)}{2-\theta\left[\lambda^{2}+(1-\lambda)^{2}\right]}
$$

is viewed as a function of $\lambda$, which is such that

$$
\frac{\partial \varepsilon(\lambda)}{\partial \lambda}>0
$$

Clearly, $\mathcal{U}(\lambda)$ integrates the impact of urban costs and $\mathcal{T}(\lambda)$ that of transportation costs. Note that the value of $\mathcal{T}(\lambda)$ depends on the mass of varieties produced in each region. As shown by (1) and (3), $N_{r}$ depends itself on $S_{r}$, which in turn depends on the value of $\theta$.

Last, because $\omega(\varepsilon)$ is strictly increasing in $\varepsilon$ and $\varepsilon(\lambda)$ strictly increasing in $\lambda$, it is readily verified that:

$$
\frac{d \mathcal{U}(\lambda)}{d \lambda}>0 \quad \frac{d \mathcal{T}(\lambda)}{d \lambda}<0
$$

Since $\mathcal{T}=\left(w_{2} / P_{2}\right) /\left(w_{1} / P_{1}\right)$, a decrease in $\mathcal{T}$ means that region 1 becomes relatively more attractive. Therefore, the two foregoing inequalities imply that a rise in the population of region 1 strengthens both the dispersion force - associated with urban costs - and the agglomeration force - generated by transportation costs. As expected, the equilibrium share of workers located in region 1 is the outcome of these two opposite forces.

\subsection{Symmetry}

To start with, we focus on the symmetric configuration $\lambda=1 / 2$. Clearly, this configuration is always a spatial equilibrium. To study its stability, we derive the elasticity of the indirect utility in one region with respect to the number of workers in that region. Totally differentiating $V_{r}$ and evaluating the resulting expression at $\lambda=1 / 2$, we obtain

$$
\frac{d V_{r}}{V_{r}}=-\frac{\theta L_{r} / 2}{1-\theta L_{r} / 2} \frac{d L_{r}}{L_{r}}+\frac{d w_{r}}{w_{r}}-\frac{d P_{r}}{P_{r}} \quad r=1,2 .
$$

Let

$$
Z \equiv \frac{1-T^{1-\sigma}}{1+T^{1-\sigma}} \in(0,1)
$$


Then, the wage equations imply

$$
\left.\left(\frac{\sigma}{Z}-1\right) \frac{d w_{r}}{w_{r}}\right|_{\lambda=1 / 2}=\left.(\sigma-1) \frac{d P_{r}}{P_{r}}\right|_{\lambda=1 / 2}+\left.\frac{d S_{r}}{S_{r}}\right|_{\lambda=1 / 2} \quad r=1,2 .
$$

Similarly, totally differentiating the price indices yields

$$
\left.\left(\frac{1-\sigma}{Z}\right) \frac{d P_{r}}{P_{r}}\right|_{\lambda=1 / 2}=\left.(1-\sigma) \frac{d w_{r}}{w_{r}}\right|_{\lambda=1 / 2}+\left.\frac{d S_{r}}{S_{r}}\right|_{\lambda=1 / 2} \quad r=1,2 .
$$

Solving (6) and (7) for $d w_{r} / w_{r}$ and $d P_{r} / P_{r}$, we get

$$
\begin{aligned}
& \left.\frac{d w_{r}}{w_{r}}\right|_{\lambda=1 / 2}=\left.\frac{Z}{\sigma(Z+1)-Z} \frac{d S_{r}}{S_{r}}\right|_{\lambda=1 / 2} \quad r=1,2 \\
& \left.\frac{d P_{r}}{P_{r}}\right|_{\lambda=1 / 2}=-\left.\frac{\sigma Z}{(\sigma-1)[\sigma(Z+1)-Z]} \frac{d S_{r}}{S_{r}}\right|_{\lambda=1 / 2} \quad r=1,2
\end{aligned}
$$

where

$$
\frac{d S_{r}}{S_{r}}=\frac{1-\theta L_{r}}{1-\theta L_{r} / 2} \frac{d L_{r}}{L_{r}} \quad r=1,2
$$

from (1). Inserting (8), (9), and (10) into (4), we have the elasticity of the indirect utility at $\lambda=1 / 2$ :

$$
\left.\frac{L_{r}}{V_{r}} \frac{d V_{r}}{d L_{r}}\right|_{\lambda=1 / 2}=\frac{4-2 \theta}{4-\theta}\left[\frac{(2 \sigma-1) Z}{(\sigma-1)[\sigma(Z+1)-Z]}-\frac{\theta}{2(2-\theta)}\right] .
$$

This expression will allow us to prove the following property.

Proposition 2. The symmetric equilibrium is stable if and only if

$$
\Omega(Z) \equiv \frac{(2 \sigma-1) Z}{(\sigma-1)[\sigma(Z+1)-Z]}<\frac{\theta}{2(2-\theta)} \equiv \Gamma(\theta) .
$$

Proof. It follows immediately from (11) that

$$
\left.\frac{L_{r}}{V_{r}} \frac{d V_{r}}{d L_{r}}\right|_{\lambda=1 / 2}<0 \Longleftrightarrow \frac{(2 \sigma-1) Z}{(\sigma-1)[\sigma(Z+1)-Z]}<\frac{\theta}{2(2-\theta)} .
$$

Noting that $\partial \Omega(Z) / \partial Z>0, \partial Z / \partial T>0$ and $\partial \Gamma(\theta) / \partial \theta>0$, we are able to derive the analytical expression for the break point in terms of transportation costs, called the $T$-break point, at which the symmetric equilibrium becomes unstable. 
Proposition 3. If $\theta \in\left(0, \min \left\{\frac{4}{\sigma+1}, 1\right\}\right)$, then there exists a unique T-break point given by

$$
T^{b}=\left\{\frac{(2 \sigma-1)[1-(\sigma-1) \Gamma(\theta)]}{(2 \sigma-1)+(\sigma-1) \Gamma(\theta)}\right\}^{\frac{1}{1-\sigma}}
$$

and the symmetric configuration is a stable equilibrium if and only if $T<T^{b}$. However, there exists no T-break point and the symmetric equilibrium is always stable regardless of transportation costs if and only if $\sigma>3$ and $\theta \in\left[\frac{4}{\sigma+1}, 1\right)$.

Proof. We know $\partial \Omega(Z) / \partial Z>0$ and $Z \in(0,1)$. This implies $\Omega(Z) \in\left(0, \frac{1}{\sigma-1}\right)$. Thus, if $\Gamma(\theta) \in\left(0, \frac{1}{\sigma-1}\right)$ or, equivalently as $\theta<1$, if $\theta \in\left(0, \min \left\{\frac{4}{\sigma+1}, 1\right\}\right)$, we have a unique value of $Z$ that satisfies

$$
\left.\frac{L_{r}}{V_{r}} \frac{d V_{r}}{d L_{r}}\right|_{\lambda=1 / 2}=0
$$

(or $\Omega(Z)=\Gamma(\theta)$ ). Since $\partial Z / \partial T>0$, there exists a unique value of $T$, denoted by $T^{b}$, such that (13) holds. Solving (13) for $T$, we have the desired expression for $T^{b}$. In addition, $T<T^{b}$ implies

$$
\left.\frac{L_{r}}{V_{r}} \frac{d V_{r}}{d L_{r}}\right|_{\lambda=1 / 2}<0
$$

so that the symmetric configuration is stable; and vice versa.

If $\sigma>3$ and $\theta \in\left[\frac{4}{\sigma+1}, 1\right)$, we have

$$
\left.\frac{L_{r}}{V_{r}} \frac{d V_{r}}{d L_{r}}\right|_{\lambda=1 / 2}<0
$$

for all transportation costs $T \in(1, \infty)$. In this case, there exists no $T$-break point so that the symmetric configuration is always a stable equilibrium.

When varieties are fairly close substitutes $(\sigma>3)$, the benefit of a better access to all varieties is small. Hence, when commuting costs are sufficiently large $(\theta>4 /(\sigma+$ $1)$ ), the symmetric configuration is always stable, unlike what we observe in the standard core-periphery model. By contrast, when one of these two conditions does not hold, the symmetric configuration may become unstable. Indeed, despite the fact that the whole range of varieties in the economy shrinks when workers are agglomerated (Proposition 1), they benefit from the access to a wider array of local varieties. When transportation costs are high $\left(T \geq T^{b}\right)$, the net benefit of having all varieties locally produced is sufficiently large to outweigh the higher urban costs that workers must bear by being agglomerated. As shown by Proposition 3, this is so if varieties are sufficiently differentiated $(\sigma \leq 3)$, or if commuting costs are sufficiently low $(\theta \leq 4 /(\sigma+1))$, or both. 
Alternatively, we can derive the break point in terms of commuting costs, which we call the $\theta$-break point.

Proposition 4. If $\sigma>3$, or if both $\sigma \leq 3$ and

$$
T<\bar{T} \equiv\left[\frac{(2 \sigma-1)(3-\sigma)}{5 \sigma-3}\right]^{\frac{1}{1-\sigma}} \in(1, \infty),
$$

then there exists a unique $\theta$-break point given by

$$
\theta^{b}=\frac{4(2 \sigma-1) Z}{\left(\sigma^{2}+2 \sigma-1\right) Z+\sigma(\sigma-1)}
$$

and the symmetric configuration is a stable equilibrium if and only if $\theta>\theta^{b}$. If both $\sigma \leq 3$ and $T \geq \bar{T}$, there exists no $\theta$-break point and the symmetric equilibrium is always unstable regardless of commuting costs.

Proof. Solving

$$
\left.\frac{L_{r}}{V_{r}} \frac{d V_{r}}{d L_{r}}\right|_{\lambda=1 / 2}=0
$$

(or $\Omega(Z)=\Gamma(\theta)$ ) for $\theta$, we get the foregoing expression for $\theta^{b}$. Since $\theta^{b}$ is increasing in $Z$ and $Z \in(0,1)$, it follows that $\theta^{b}$ belongs to $\left(0, \frac{4}{\sigma+1}\right)$. If $\sigma>3$, then $\theta^{b}<1$ regardless of the value of $T$. Because

$$
\left.\frac{L_{r}}{V_{r}} \frac{d V_{r}}{d L_{r}}\right|_{\lambda=1 / 2}<0
$$

when $\theta>\theta^{b}$, the symmetric configuration is stable; and vice versa.

By contrast, when $\sigma \leq 3$, there exists a single value

$$
\bar{Z}=\frac{\sigma(\sigma-1)}{-\sigma^{2}+6 \sigma-3} \in(0,1]
$$

such that $\theta^{b}<1$ if and only if $Z<\bar{Z}$. Using (5) then yields the upper bound $\bar{T}$ on $T$. Hence, there is no $\theta$-break point if and only if $T \geq \bar{T}$. Finally, when $\sigma \leq 3$ and $T<\bar{T}$, it is readily verified that the $\theta$-break point is unique and given by $\theta^{b}<1$.

Note that (14) is the reciprocal relationship of (12). In addition, at the border value $\sigma=3$, the domain $\left[\frac{4}{\sigma+1}, 1\right)$ is empty whereas $\bar{T}$ goes to infinity. Accordingly, Proposition 4 may be viewed as the counterpart of Proposition 3 in terms of commuting costs. 


\subsection{Agglomeration}

We now come to the case of an agglomeration $(\lambda=1)$. Then, the price indices yield the relationship

$$
P_{2}=T P_{1}
$$

Similarly, from the wage equations, we have

$$
w_{2}=T^{\frac{1-\sigma}{\sigma}} w_{1}
$$

These two relationships imply that the ratio of indirect utilities is given by

$$
\left.\frac{V_{2}}{V_{1}}\right|_{\lambda=1}=\frac{T^{\frac{1-2 \sigma}{\sigma}}}{1-\theta / 2} .
$$

Using this relationship, the sustainability of agglomeration as a spatial equilibrium can be obtained under the following conditions.

Proposition 5. Agglomeration is a spatial equilibrium if and only if $T>(1-\theta / 2)^{\frac{\sigma}{1-2 \sigma}}$.

Proof. From (15), we have

$$
\left.\frac{V_{2}}{V_{1}}\right|_{\lambda=1}<1 \Longleftrightarrow T>(1-\theta / 2)^{\frac{\sigma}{1-2 \sigma}} .
$$

The analytical expression of the $T$-sustain point may then be obtained as follows.

Proposition 6. The T-sustain point is given by

$$
T^{s}=(1-\theta / 2)^{\frac{\sigma}{1-2 \sigma}}
$$

and agglomeration is a stable equilibrium if and only if $T>T^{s}$.

Proof. Because

$$
\left.\frac{V_{2}}{V_{1}}\right|_{\lambda=1}=1
$$

must hold at the $T$-sustain point, we obtain the desired expression.

This proposition confirms the numerical simulations provided by Helpman [7]. In fact, our result is even stronger than Helpman's because the array of varieties available in the 
agglomeration is narrower than what it is under dispersion. Our result can be understood as follows. Workers are willing to bear the high urban costs associated with their agglomeration within a single city as well as the consumption of a narrower range of varieties because buying varieties from the other region is very expensive.

In the same vein, the $\theta$-sustain point is derived below.

Proposition 7. If $T \in\left(1,2^{\frac{\sigma}{2 \sigma-1}}\right)$, then there exists a unique $\theta$-sustain point given by

$$
\theta^{s}=2\left(1-T^{\frac{1-2 \sigma}{\sigma}}\right)
$$

and agglomeration is a stable equilibrium if and only if $\theta<\theta^{s}$. If $T \in\left[2^{\frac{\sigma}{2 \sigma-1}}, \infty\right)$, then agglomeration is sustainable for all commuting costs.

Proof. Solving $V_{2} / V_{1}=1$ for $\theta$, we get the foregoing expression. Because $\theta^{s}$ must be smaller than 1 , we get the second part of the claim.

Note that (17) is the reciprocal of (16) so that Proposition 7 is the reciprocal of Proposition 6 in terms of commuting costs.

\subsection{Break point versus sustain point}

As in Fujita et al. [6], the sustain point does not coincide with the break point in our model. It is, therefore, worthwhile to examine the relative magnitude of these points. ${ }^{5}$

Proposition 8. Assume that a T-break point exists. Then, the T-sustain point is smaller than the T-break point.

Proof. Consider the ratio of the $T$-sustain and $T$-break points. Taking the limit of this ratio when $\theta$ tends to zero, we have

$$
\lim _{\theta \rightarrow+0} \frac{T^{s}}{T^{b}}=1
$$

We now show that the ratio decreases as $\theta$ rises from 0 . Differentiating the ratio with respect to $\theta$ yields

$$
\operatorname{sgn}\left\{\frac{\partial}{\partial \theta}\left(\frac{T^{s}}{T^{b}}\right)\right\}=\operatorname{sgn} \Phi(\theta, \sigma)
$$

where $\Phi(\theta, \sigma) \equiv-8 \sigma^{2}+(\sigma+1)(3 \sigma-1) \theta$. We know that $\theta$ must be lower than $\min \{4 /(\sigma+1), 1\}$ for a $T$-break point to exist. Clearly, $\Phi(\theta, \sigma)$ is increasing in $\theta$. Because both $\Phi\left(\frac{4}{\sigma+1}, \sigma\right)=$

\footnotetext{
${ }^{5}$ Note that Robert-Nicoud [14] analyzes the relative magnitude of these two points for a family of economic geography models. Unfortunately, our model does not belong to this family, thus implying that we cannot use his results.
} 
$-4(2 \sigma-1)(\sigma-1)<0$ and $\Phi(1, \sigma)=-5 \sigma^{2}+2 \sigma-1<0$, it must be that $\Phi(\theta, \sigma)<0$ for all $\theta<\min \{4 /(\sigma+1), 1\}$. Thus, $T^{s} / T^{b}<1$ for all $\theta \in(0, \min \{4 /(\sigma+1), 1\})$.

Proposition 9. When they exist, the $\theta$-sustain point is larger than the $\theta$-break point.

Proof. Assume that $\sigma \leq 3$ or that $\sigma>3$ and $\theta \in(0,4 /(\sigma+1))$. Then, for any admissible $\theta$, there exists a break point in terms of transportation costs. In turn, Proposition 8 implies that $T^{b}>T^{s}$ for any admissible $\theta$. From Propositions 4 and 7 , it follows that $\theta^{b}$ and $\theta^{s}$ are increasing in $T$. By construction, $\theta^{b}$ is the reciprocal of (12), whereas $\theta^{s}$ is the reciprocal of (16). Consequently, it must be that $\theta^{s}>\theta^{b}$ for any $T$. Indeed, $\theta^{s} \leq \theta^{b}$ would imply $T^{b} \leq T^{s}$ for some $\theta$, thus contradicting Proposition 8 .

Consider now the case where $\sigma>3$ and $\theta \in[4 /(\sigma+1), 1)$. The curve (14) is always strictly below the horizontal line at $\theta$, whereas the curve (17) intersects this line once because $T^{s}$ always exists and is unique. Hence, it must be that $\theta^{s}>\theta^{b}$ for any $T$.

The foregoing results are somewhat reminiscent of those derived in standard models of economic geography. However, there are major and striking differences. First, in accord with Helpman's [7] simulations, agglomeration is a stable equilibrium when transportation costs are sufficiently large. However, as in Anas [2], a steady decrease in transportation costs always leads to the dispersion of the industry. Second, agglomeration is always a stable equilibrium once commuting costs are low enough. Furthermore, once transportation costs take low values, agglomeration necessarily arises provided that commuting costs are themselves sufficiently low. Note also that agglomeration may even arise under intermediate or large commuting costs when transportation costs are sufficiently large.

[Insert Figure 1 about here]

Third, whereas there always exists a $T$-sustain point, a $T$-break point may not exist in our model. In Figure 1a, drawn for $\sigma \leq 3$, a $T$-break point exists because the curve $\theta^{b}(T)$, given by the function (14), intersects once the horizontal line at $\theta=1$. By contrast, in Figure 1b drawn for $\sigma>3$, this curve has a horizontal asymptote at $\theta=4 /(\sigma+1)<1$. Hence, it does not intersect the horizontal line at $\theta=1$, implying that there exists no $T$-break point as long as $4 /(\sigma+1) \leq \theta<1$. Consider now the curve $\theta^{s}(T)$ defined by the function (17). In both figures, this curve intersects the horizontal line at $\theta=1$, so that a $T$ sustain point always exists. More surprisingly maybe, the existence of a $\theta$-break point and of a $\theta$-sustain point is not guaranteed. Hence, the relationship between transportation costs and commuting costs is not necessarily one-to-one over the domain $(T, \theta) \in(1, \infty) \times(0,1)$.

Last, unlike Fujita et al. [6] and others, we have been able to derive the explicit analytical expressions for both the T-break and T-sustain points. Note that we can also determine 
the $\theta$-break and $\theta$-sustain points (when they exist). This will allow us to describe below the interplay between these two types of costs in the formation of the space-economy.

Observe that agglomeration is sustainable in the region below $\theta^{s}(T)$, whereas dispersion is a stable equilibrium in the region above $\theta^{b}(T)$. As a result, in the region situated between the two curves, there exist at least three stable equilibria. Unfortunately, as mentioned in the introduction, we have not been able to provide a full characterization of the set of equilibria. This is why, in the next section, we appeal to numerical solutions.

\subsection{The set of equilibria: numerical examples}

The market outcome is driven by three main parameters: (i) the commuting cost, $\theta>$ 0, (ii) the elasticity of substitution between differentiated goods, $\sigma>1$, and (iii) the transportation costs, $T>1$. In order to illustrate the role of the four points discussed in the foregoing, we impose the restriction $\sigma \leq 3$, which implies that $\theta$ may take any value in $(0,1)$.

Figure 2 is depicted for $\theta=0.200$ and $\sigma=2.5$ so that $T^{s}=1.068$ and $T^{b}=1.074$. Transportation costs take three possible values: $T=1.090, T=1.072$ (which will be selected in the next experiment on commuting costs) and $T=1.050$. In the first case (Figure 2a), there are three equilibria, but dispersion is unstable while full agglomeration within a single city is the only stable equilibrium as $T$ is larger than $T^{b}$. In the second (Figure $2 \mathrm{~b}$ ), there exist five equilibria: the two (mirror) equilibria involving partial agglomeration in two cities of unequal size are unstable, whereas the other three equilibria, corresponding to dispersion or full agglomeration, are stable because the value of $T$ belongs to $\left[T^{s}, T^{b}\right]$. In the last case (Figure 2c), the only equilibrium involves two cities of equal size and is stable, $T$ being smaller than $T^{s}$. Such a pattern concurs with what Krugman [9] have obtained in the core-periphery model, except that the sequence of configurations is reversed.

In Krugman [9], the only cost generated by the formation of an agglomeration is related to the provision of the manufactured goods to the - by assumption - immobile farmers residing in the periphery. Here, agglomerating firms give rise to specific costs, i.e. higher urban costs and a narrower range of varieties, which workers are willing to bear provided that shipping the manufactured goods between regions is expensive. Hence, when trans-

portation costs are low, workers are better off by being dispersed. Accordingly, we may conclude that the interplay of agglomeration and dispersion forces changes with the nature of the forces at work.

[Insert Figure 2 about here]

Figure 3 is depicted for $T=1.072$ (which has been chosen above) and $\sigma=2.5$ so that $\theta^{b}=0.189$ and $\theta^{s}=0.205$. Commuting costs are allowed to take the following values: 
$\theta=0.220, \theta=0.200$ (which has also been selected in the foregoing experiment) and $\theta=0.180$. In the first case (Figure 3a), the only stable equilibrium involves two cities of equal size. In the second (Figure 3b), there exist five equilibria in which dispersion and full agglomeration are the only ones that are stable as long as $\theta$ belongs to $\left[\theta^{b}, \theta^{s}\right]$. In the last case (Figure 3c), full agglomeration is the only stable equilibrium. Again, a pattern similar to the one derived in the core-periphery model emerges. However, the role of the two spatial costs is reversed: low commuting costs instead of high transportation costs foster agglomeration, and vice versa.

[Insert Figure 3 about here]

\section{Concluding remarks}

This paper has provided a simple and unified treatment of the interactions between the transportation costs of goods and the commuting costs borne by workers. This is best shown by comparing the values of workers' welfare reached at each of the two equilibrium configurations. Indeed, we have

$$
V^{A}=\left(N^{A}\right)^{\frac{\sigma}{\sigma-1}} \quad V^{D}=\left(N^{D}\right)^{\frac{\sigma}{\sigma-1}}\left(\frac{1+T^{1-\sigma}}{2}\right)^{\frac{1}{\sigma-1}}
$$

where the superscript $A$ (resp. $D)$ stands for agglomeration (resp. dispersion). Consequently, by being agglomerated, workers save on the transportation costs of the differentiated product, but have access to a narrower range of varieties. By contrast, by being dispersed, workers have access to a broader range of varieties, but must then bear the cost of shipping the varieties produced in the other city. The equilibrium outcome shows how the market solves this trade-off.

Using a Dixit-Stiglitz-iceberg framework, we have also been able to determine the four threshold values corresponding to the break and sustain points, and to uncover the relationships between them. Our analysis makes it clear that what really matters for the structure of the space-economy is not just the level of economic integration, but the interplay between transportation costs and urban costs.

It is worth noting that our model has proven to be easy to handle, while retaining most general equilibrium effects. This suggests that it can be used as a building-block in a more general setting, such as growth models with infinitely-lived consumers or overlapping generations. This, in turn, should permit the study of the long-run impact of urban and transportation costs on the structure, size and number of cities in a dynamic context.

\section{References}

[1] W. Alonso, Location and Land Use, Harvard University Press, Cambridge 1964. 
[2] A. Anas, Vanishing cities: What does the new economic geography imply about the efficiency of urbanization?, Journal of Economic Geography 4 (2004) 181-199.

[3] P. Bairoch, Cities and Economic Development: From the Dawn of History to the Present, University of Chicago Press, Chicago 1988.

[4] A.K. Dixit, J.E. Stiglitz, Monopolistic competition and optimum product diversity, American Economic Review 67 (1977) 297-308.

[5] G. Duranton, D. Puga, Nursery cities: Urban diversity, process innovation, and the life cycle of products, American Economic Review 91 (2001) 1454-1477.

[6] M. Fujita, P. Krugman, A.J. Venables, The Spatial Economy: Cities, Regions, and International Trade, MIT Press, Cambridge 1999.

[7] E. Helpman, The size of regions, in: D. Pines, E. Sadka, I. Zilcha (Eds.), Topics in Public Economics, Cambridge University Press, Cambridge 1998, pp. 33-54.

[8] P. Hohenberg, L.H. Lees, The Making of Urban Europe (1000-1950), Harvard University Press, Cambridge 1985.

[9] P. Krugman, Increasing returns and economic geography, Journal of Political Economy 99 (1991) 483-499.

[10] P. Krugman, R. Livas Elizondo, Trade policy and the Third World metropolis, Journal of Development Economics 49 (1996) 137-150.

[11] Y. Murata, Product diversity, taste heterogeneity, and geographic distribution of economic activities: Market vs. non-market interactions, Journal of Urban Economics 53 (2003) 126-144.

[12] G.I.P. Ottaviano, T. Tabuchi, J.-F. Thisse, Agglomeration and trade revisited, International Economic Review 43 (2002) 409-435.

[13] G.I.P. Ottaviano, F. Robert-Nicoud, The 'genome' of NEG models with vertical linkages: A positive and normative synthesis, forthcoming in Journal of Economic Geography (2005).

[14] F. Robert-Nicoud, The structure of simple 'new economic geography' models, forthcoming in Journal of Economic Geography (2005).

[15] T. Tabuchi, Urban agglomeration and dispersion: A synthesis of Alonso and Krugman, Journal of Urban Economics 44 (1998) 333-351.

[16] T. Tabuchi, J.-F. Thisse, Regional specialization, urban hierarchy, and commuting costs, CORE Discussion Paper Nº60 (2003). 
Figure 1: Break and sustain points.

Figure 1a: $T$-break, $T$-sustain, $\theta$-break, and $\theta$-sustain points $(\sigma \leq 3)$.

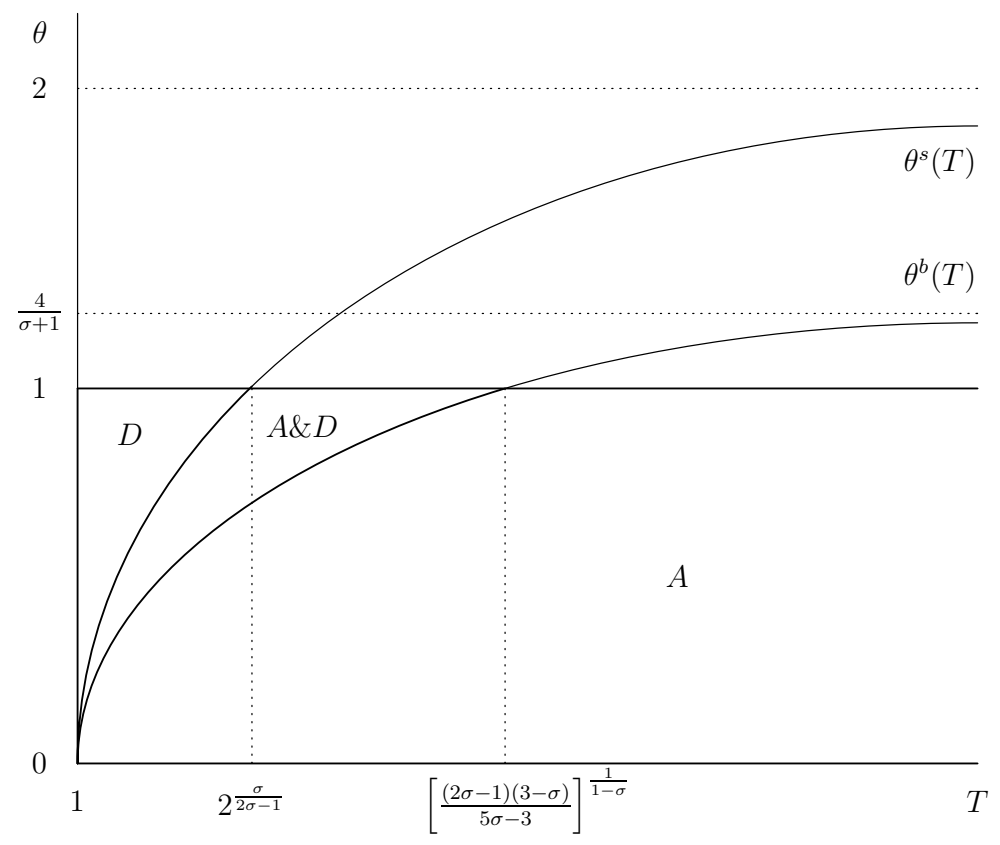

Figure 1b: $T$-break, $T$-sustain, $\theta$-break, and $\theta$-sustain points $(\sigma>3)$.

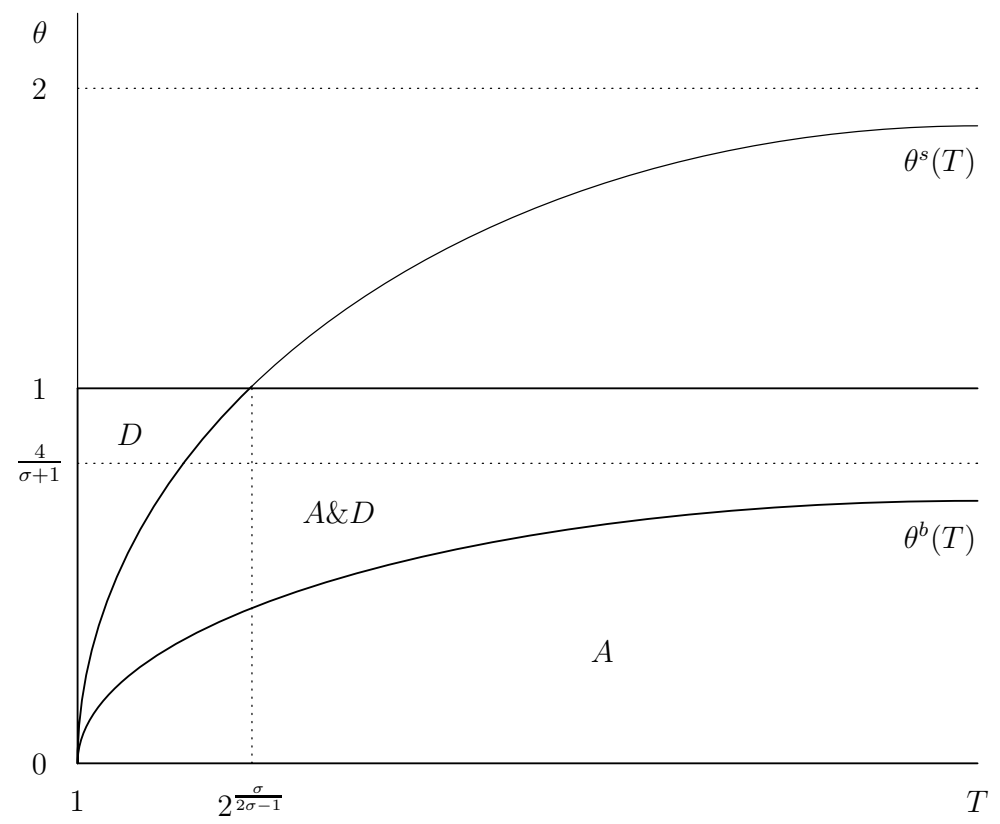

A: Agglomeration is a stable equilibrium

$D$ : Dispersion is a stable equilibrium 
Figure 2: Real wage differentials $(\theta=0.200$ and $\sigma=2.5)$.

Figure 2a: Real wage differential for $T=1.090$

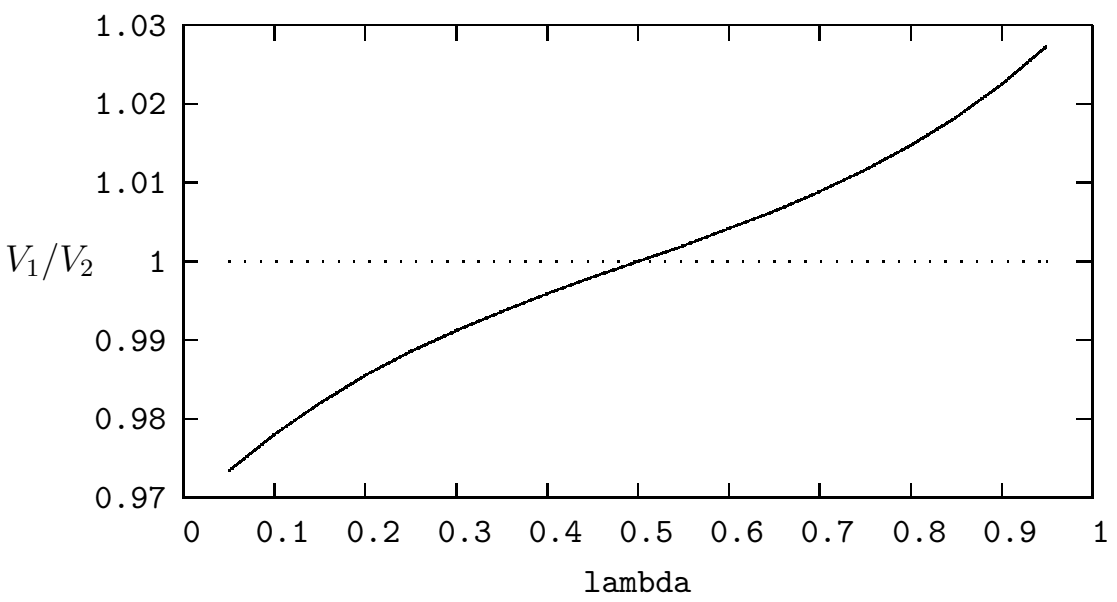

Figure 2b: Real wage differential for $T=1.072$

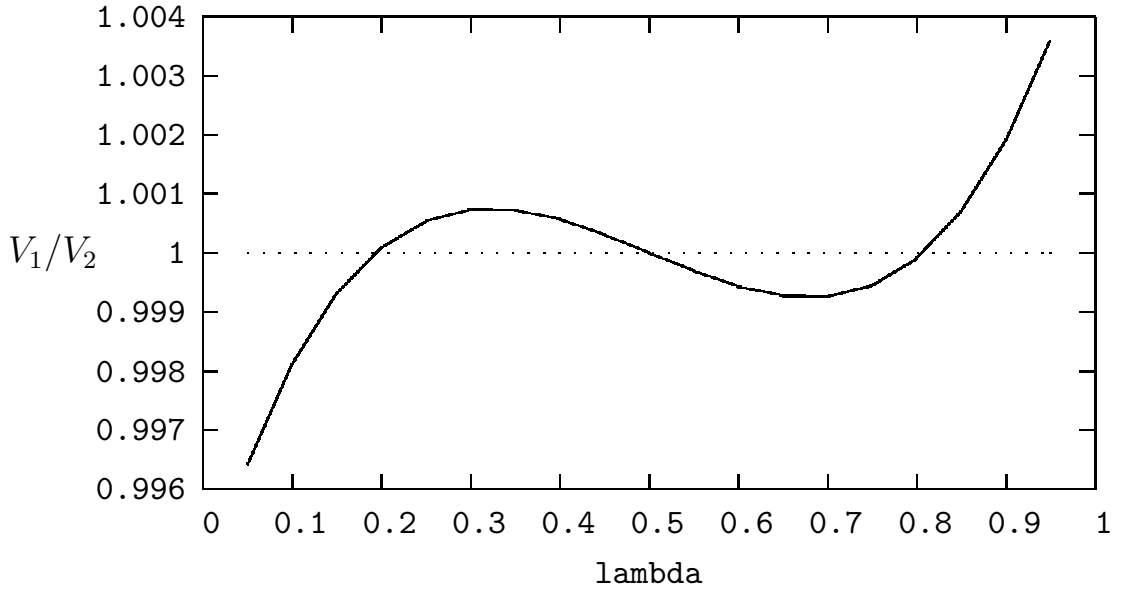

Figure 2c: Real wage differential for $T=1.050$

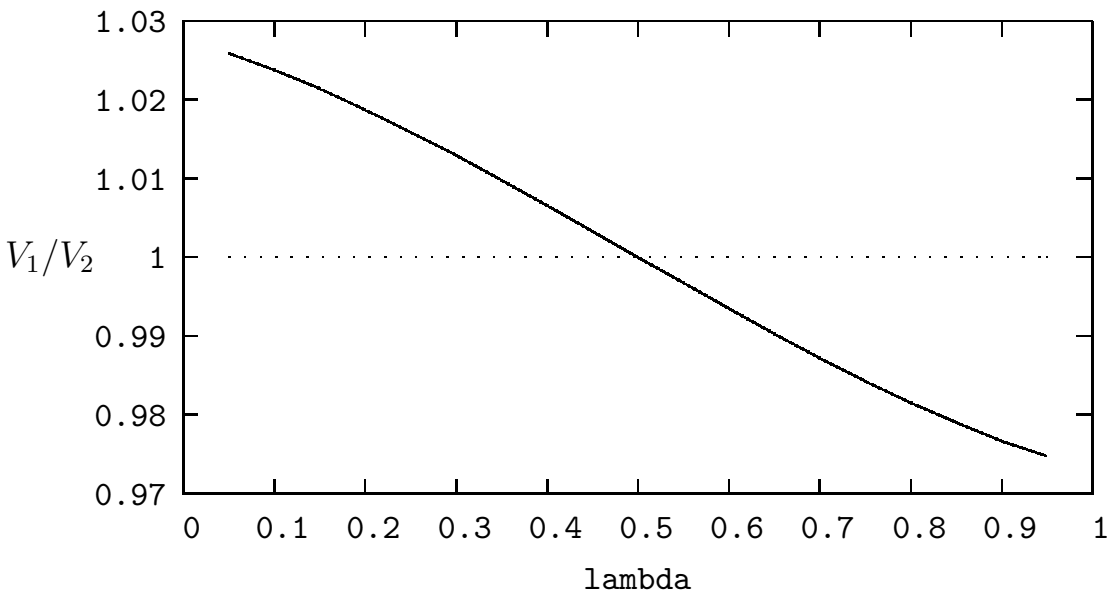


Figure 3: Real wage differentials $(T=1.072$ and $\sigma=2.5)$.

Figure 3a: Real wage differential for theta $=0.220$

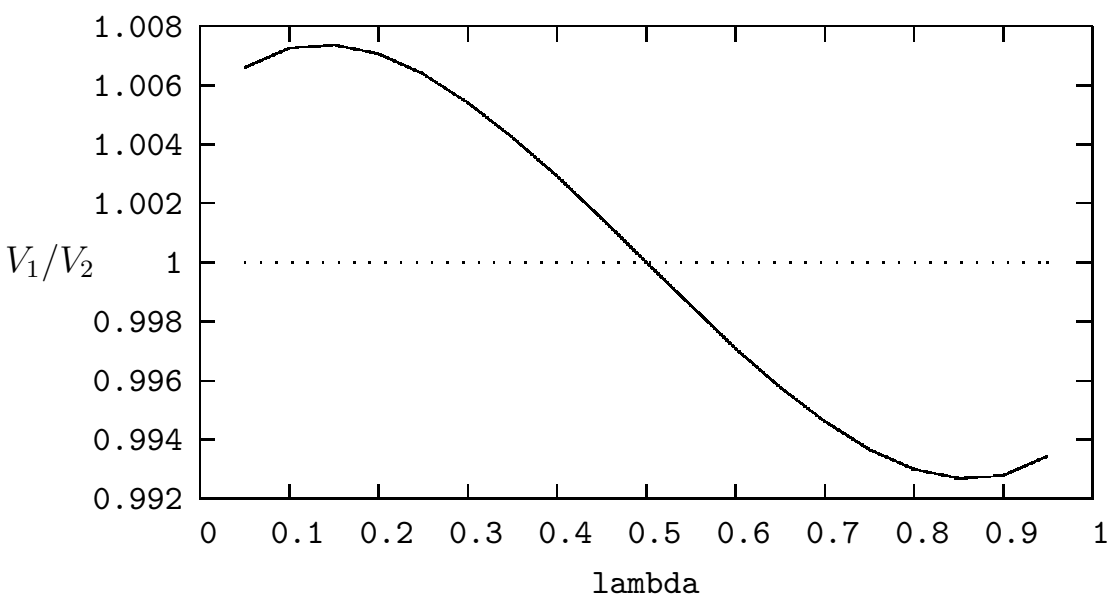

Figure 3b: Real wage differential for theta $=0.200$

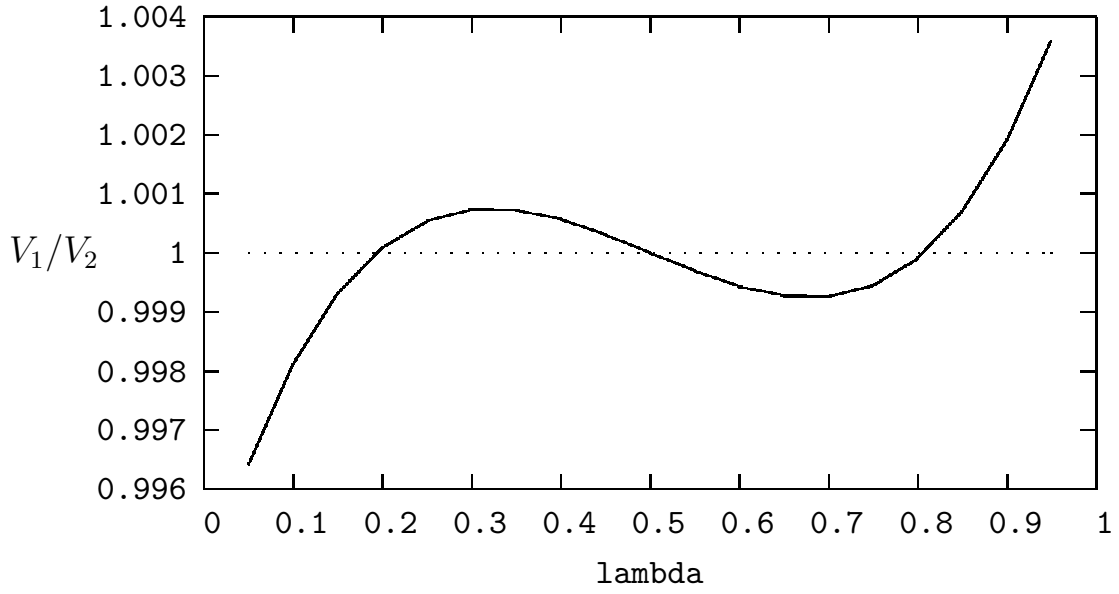

Figure 3c: Real wage differential for theta $=0.180$

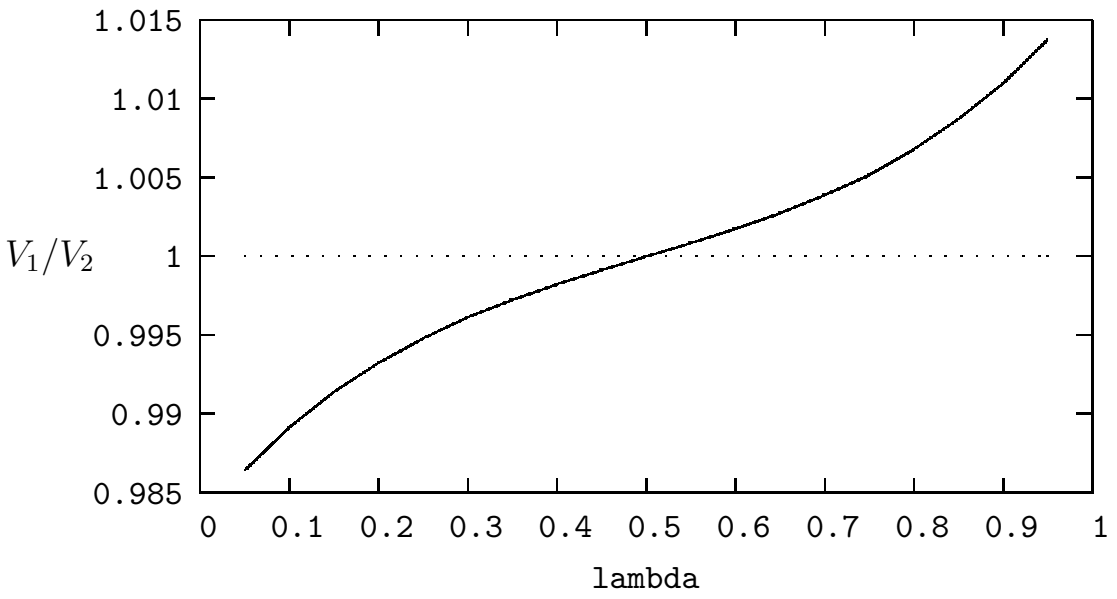


Département des Sciences Économiques de l'Université catholique de Louvain

Institut de Recherches Économiques et Sociales

Place Montesquieu, 3

1348 Louvain-la-Neuve, Belgique 